\title{
Comparison of HER2 Status Before and After Trastuzumab- based Chemotherapy in Patients With Advanced Gastric Cancer
}

\author{
TAKASHI KIJIMA ${ }^{1}$, TAKAAKI ARIGAMI ${ }^{2}$, YOSHIKAZU UENOSONO ${ }^{1}$, TSUBASA HIRAKI ${ }^{3}$, \\ SHIGEHIRO YANAGITA ${ }^{1}$, DAISUKE MATSUSHITA ${ }^{1}$, KEISHI OKUBO $^{1}$, MASATAKA SHIMONOSONO ${ }^{1}$, \\ SUMIYA ISHIGAMI ${ }^{1}$, KOSEI MAEMURA $^{1}$, AKIHIDE TANIMOTO ${ }^{3}$ and SHOJI NATSUGOE ${ }^{1,2}$ \\ ${ }^{1}$ Department of Digestive Surgery, Breast and Thyroid Surgery, \\ Kagoshima University Graduate School of Medical and Dental Sciences, Kagoshima, Japan; \\ ${ }^{2}$ Department of Bio-oncological Surgery, Kagoshima University Graduate \\ School of Medical and Dental Sciences, Kagoshima, Japan; \\ ${ }^{3}$ Department of Pathology, Kagoshima University Graduate \\ School of Medical and Dental Sciences, Kagoshima, Japan
}

\begin{abstract}
Background/Aim: The aim of the present study was to compare human epidermal growth factor receptor 2 (HER2) expression before and after trastuzumab-based chemotherapy in patients with advanced HER2-positive gastric cancer. Materials and Methods: We assessed HER2 expression using immunohistochemistry and/or fluorescence in situ hybridization in pre-treatment biopsied specimens and post-treatment resected specimens obtained from seven patients with advanced HER2-positive gastric cancer receiving trastuzumab-based chemotherapy. Results: Four patients maintained the HER2-positive status and three patients had a change in HER 2 expression from positive to negative. In patients showing the loss of HER2 expression after treatments, HER2-positive tumor cells with a dominant histological type disappeared, and HER2-negative tumor cells with another dominant histological type were identified. Conclusion: HER 2 expression can change after trastuzumabbased chemotherapy in patients with advanced HER2positive gastric cancer. Continuous monitoring of HER2 expression after treatments may be utilized to determine whether the continued use of trastuzumab is advisable.
\end{abstract}

This article is freely accessible online.

Correspondence to: Takashi Kijima, MD, Ph.D., Department of Digestive Surgery, Breast and Thyroid Surgery, Kagoshima University Graduate School of Medical and Dental Sciences, 8-351 Sakuragaoka, Kagoshima 890-8520, Japan. Tel: +81 992755361, e-mail: ta@m2.kufm.kagoshima-u.ac.jp

Key Words: HER2, advanced gastric cancer, trastuzumab, chemotherapy.
Gastric cancer is the fifth most common cancer worldwide (1). For many patients, their disease has become inoperable by the time they are diagnosed, or their disease recurs after curative resection (2). For patients with advanced, unresectable cancer, systemic chemotherapy is generally prescribed as the primary therapy (3).

Human epidermal growth factor receptor 2 (HER2) is associated with cell proliferation, apoptosis and differentiation (4). A study by Bang et al. demonstrated that HER2-targeting therapy using trastuzumab and standard chemotherapy is the standard treatment for advanced HER2-positive gastric cancer patients (5). However, a recent chemotherapy study has found that patients who responded well received surgery as a part of a multimodality treatment strategy (6). In addition, a number of reports have demonstrated that chemotherapy including trastuzumab made it possible to perform successful gastrectomy in unresectable HER2-positive patients (7-10), and some studies examining breast cancer have suggested that HER2 positivity may be lost after neoadjuvant therapy with or without trastuzumab $(11,12)$. However, few reports to date have assessed the loss of HER2 expression after trastuzumabbased chemotherapy in patients with advanced HER2-positive gastric cancer. In the present study, we investigated the expression pattern of HER2 in surgically resected specimens obtained from patients undergoing chemotherapy including trastuzumab. Moreover, we compared changes in HER2 expression status before and after treatments.

\section{Materials and Methods}

Patients. The present study received approval from the Ethics Committee of Kagoshima University, and all patients provided a written informed consent for the use and publication of their information. Fourteen patients with HER2-positive advanced/recurrent gastric cancer received trastuzumab-based chemotherapy between February 2011 and 
May 2015 at the Department of Digestive Surgery, Breast, and Thyroid Surgery, at the University of Kagoshima Hospital in Kagoshima, Japan. All patients were evaluated via blood examinations, esophagogastroduodenoscopy, computed tomography (CT), and PET/CT before starting treatment. Eleven patients were classified as stage IV based on the International Union Against Cancer (UICC) criteria of tumor-nodemetastasis (TNM) classification for gastric cancer (13), three patients were diagnosed with recurrence after gastrectomy, two patients showed lymph node metastases, and one patient showed liver metastasis. Of the 14 patients, five patients underwent radical gastrectomy because of the downstaging of their disease, one patient underwent gastrectomy because of the tumor bleeding without enough downstaging, and one patient underwent surgical resection of liver metastasis. Consequently, seven patients were enrolled in the present study. Detailed clinicopathological characteristics of the patients are shown in Table I. We used the Japanese classification of gastric carcinoma: $3^{\text {rd }}$ English edition (14) to express the dominant histological type of the tumor cells. Criteria for HER 2 positivity were defined as an immunohistochemistry (IHC) score of $3+$ or an IHC score of 2+ showing fluorescence in situ hybridization (FISH) positive results $(5,15)$. All seven patients were diagnosed as HER2-positive using tumor biopsies or tumor resection specimens prior to treatments; six patients were classified as HER2 IHC $3+$, and one patient was classified as HER2 IHC 2+ showing FISH positive results.

Treatment and histological evaluation. Combination chemotherapy including trastuzumab consisted of three different regimens, as follows: 1) a capecitabine plus modified cisplatin (mXP) regimen, using a 3-week course of capecitabine $\left(2000 \mathrm{mg} / \mathrm{m}^{2} /\right.$ day $)$ taken orally on days $1-14$, with cisplatin $\left(60 \mathrm{mg} / \mathrm{m}^{2}\right)$ given intravenously on day $1 ; 2)$ a paclitaxel with S-1 $(\mathrm{S} 1+\mathrm{PTX})$ regimen, using a 4-week course of paclitaxel $\left(120 \mathrm{mg} / \mathrm{m}^{2}\right)$ given intravenously on days 1 and 15 , and TS-1 $\left(80 \mathrm{mg} / \mathrm{m}^{2}\right)$ taken orally on days $1-14$; and 3) a docetaxel, cisplatin, and S-1 (DCS) regimen, using a 4-week course of docetaxel $\left(40 \mathrm{mg} / \mathrm{m}^{2}\right)$ given intravenously on day 1 , cisplatin $\left(60 \mathrm{mg} / \mathrm{m}^{2}\right)$ given intravenously on day 1 , and TS-1 $\left(80 \mathrm{mg} / \mathrm{m}^{2}\right)$ taken orally on days 1 14. A 3-week course of trastuzumab $(8 \mathrm{mg} / \mathrm{kg}$ loading dose followed by $6 \mathrm{mg} / \mathrm{kg}$ ) was given intravenously on day 1 to every regimen. Histological response evaluation of resected tumors was performed using the Japanese classification of gastric carcinoma $3^{\text {rd }}$ English edition (14), and tumors were classified as grades $0,1 \mathrm{a}, 1 \mathrm{~b}, 2$, or 3 with the following criteria: grade 0 , no evidence of treatment effect; grade 1a, viable tumor cells remain in more than $2 / 3$ of the tumor area; grade $1 \mathrm{~b}$, viable tumor cells remain in more than $1 / 3$, but less than $2 / 3$ of the tumor area; grade 2 , viable tumor cells remain in less than $1 / 3$ of the tumor area; grade 3 , no viable tumor cells.

Immunohistochemistry. Pre-treatment biopsied specimens and posttreatment resected specimens were used for IHC analysis. All paraffin-embedded specimens were cut in $4 \mu \mathrm{m}$ sections using a conventional histological technique and transferred to a slide. Immunohistochemical staining was performed using a Hercept test kit (Dako, Carpinteria, CA, USA) according to the manufacturer's protocol using an automatic immunostainer (Dako). Staining intensity was evaluated using the 0 to $3+$ scale according to the Hercept test scoring criteria (15). In surgical specimens, a score of 0 was determined if there was no reactivity or membranous reactivity in $<10 \%$ of tumor cells. A score of $1+$ was determined if faint or barely perceptible membranous reactivity occurred in $\geq 10 \%$ of tumor cells, and the cells were reactive only in part of the membrane. A
Table I. Clinicopathological characteristics.

\begin{tabular}{ll}
\hline Characteristics & $\mathrm{n}$ \\
\hline Gender & \\
Male & 5 \\
Female & 2 \\
Age (range) & $59.9(42-70)$ \\
Histological type before treatments & \\
tub1 & 2 \\
tub2 & 2 \\
por & 3 \\
Metastatic site & \\
Lymph node & 4 \\
Liver & 3 \\
Peritoneum & 2 \\
Lung & 1 \\
Combined chemotherapy & \\
S1+PTX & 3 \\
DCS & 3 \\
mXP & 1 \\
Surgical method & \\
Distal gastrectomy & 3 \\
Total gastrectomy & 2 \\
Proximal gastrectomy & 1 \\
Hepatectomy & 1 \\
\hline
\end{tabular}

tub1: Well differentiated adenocarcinoma; tub2: moderately differentiated adenocarcinoma; por: poorly differentiated adenocarcinoma; PTX: paclitaxel; DCS: docetaxel, cisplatin plus S-1; mXP: modified capecitabine plus cisplatin.

score of $2+$ was determined if weak to moderate complete or basolateral membranous reactivity occurred in $\geq 10 \%$ of tumor cells. A score of $3+$ was determined if moderate to strong, complete or basolateral membranous reactivity occurred in $\geq 10 \%$ of tumor cells. In biopsy specimens, when membrane reactivities were observed in at least one cancer cell cluster ( $\geq 5$ cells), scores of $1+, 2+$, and $3+$ were determined, regardless of the percentage of immunoreactive area. The IHC scoring was performed by an experienced pathologist (T.H), who was blinded to the clinical details of individual patients.

Fluorescence in situ hybridization. FISH was performed using the Abbott PathVysion HER2 DNA Probe Kit protocol (Abbott Laboratories, Abbott Park, Des Plaines, IL, USA) according to the manufacturer's instructions. HER2 gene amplification status was evaluated by counting the HER 2 and CEP17 signals in 20 adjacent interphase tumor cell nuclei that were examined with fluorescent microscopes and appropriate filters. A HER2:CEP17 ratio of $\geq 2.0$ was considered positive for HER2 amplification.

\section{Results}

Trastuzumab-based chemotherapy and histological responses. Table II lists the results of the present study in detail. The mean cycle number of trastuzumab therapy before the operation was 7.6 (range $=1-16$, median: 8 ). Two patients treated a single time with trastuzumab therapy were also treated three to four times with DCS combination 
Table II. HER 2 expression before and after trastuzumab-based chemotherapy.

\begin{tabular}{|c|c|c|c|c|c|c|c|c|c|c|}
\hline \multirow[t]{2}{*}{ Case } & \multirow[b]{2}{*}{$\begin{array}{l}\text { Primary/ } \\
\text { recurrence }\end{array}$} & \multicolumn{3}{|c|}{ Before treatments } & \multicolumn{3}{|c|}{ After treatments } & \multirow[b]{2}{*}{$\begin{array}{l}\text { Therapeutic } \\
\text { response }\end{array}$} & \multicolumn{2}{|c|}{ Recurrence } \\
\hline & & $\begin{array}{l}\text { HER2 } \\
\text { IHC }\end{array}$ & $\begin{array}{l}\text { Histological } \\
\text { type }\end{array}$ & $\begin{array}{c}\text { HER2 } \\
\text { positive } \\
\text { cells }\end{array}$ & $\begin{array}{c}\text { HER2 } \\
\text { IHC }\end{array}$ & $\begin{array}{c}\text { Histological } \\
\text { type }\end{array}$ & $\begin{array}{l}\text { HER2 } \\
\text { positive } \\
\text { cells }\end{array}$ & & Site & $\begin{array}{c}\text { HER2 } \\
\text { IHC }\end{array}$ \\
\hline 1 & Primary & $3+$ & tub2 & tub2 & $3+$ & tub2>por & tub2, por & $1 b$ & Brain & $3+$ \\
\hline 2 & Primary & $3+$ & por $>$ sig $>$ tub2 & tub2 & $3+$ & por>tub2>sig & tub2 & 1a & Peritoneum & - \\
\hline 3 & Primary & $3+$ & por & por & $3+$ & por & por & $1 \mathrm{a}$ & Liver & - \\
\hline 4 & Primary & $2+(\mathrm{FISH}+)$ & tub1 & tub1 & $2+(\mathrm{FISH}+)$ & tub1>tub2 & tub1, tub2 & 0 & Lymph Node & - \\
\hline 5 & Primary & $3+$ & tub2>por & tub2 & 0 & por & - & 0 & Skin & 0 \\
\hline 6 & Primary & $3+$ & por $>$ tub2>tub1 & por & 0 & tub1>muc & - & $1 \mathrm{a}$ & No Recurrence & - \\
\hline 7 & Recurrence & $3+$ & tub1>por & tub1 & 0 & por & - & $1 \mathrm{~b}$ & Lung & - \\
\hline
\end{tabular}

tub1: Well differentiated adenocarcinoma; tub2: moderately differentiated adenocarcinoma; por: poorly differentiated adenocarcinoma; sig: signetring cell carcinoma; muc: mucinous adenocarcinoma.

therapy. Out of a total of seven patients, two patients were found to have a histological grade of $1 \mathrm{~b}$, three patients had a grade of $1 \mathrm{a}$, and two patients had a grade of 0 .

\section{HER2 expression and pathological change in resected specimens} after trastuzumab-based chemotherapy. Before treatment, six patients were classified as HER2 IHC 3+, and one patient was classified as IHC $2+$ showing FISH positive results. We observed a change in the HER2 score of resected specimens after trastuzumab-based chemotherapy; three patients were classified as IHC $3+$, one patient was classified as IHC $2+$ showing FISH positive results, and three patients were classified as IHC 0 . While HER2-positive tumor cells were the dominant histological type in six out of seven patients before treatment, three of the seven patients (42.9\%) showed negative HER2 expression in resected specimens after trastuzumab-based chemotherapy. Based on the HER2 status after treatment, we divided patients into two groups: the maintained HER2 expression group and the loss of HER2 expression group. There was no change in the dominant histological type for any of the four patients in the maintained HER 2 expression group (Figure 1a and $b$ ). For the three patients in the loss of HER2 expression group, HER2-positive tumor cells with the dominant histological type identified before treatment disappeared, but HER2-negative tumor cells with another dominant histological type appeared (Figure 1c and d).

Recurrence. All four patients in the maintained HER2 expression group had a recurrence at 3.4-8.8 months after gastrectomy (average: 6.2 months). One patient had a brain metastasis 8.8 months after gastrectomy, and brain tumors were surgically removed; HER2 expression in the brain metastasis was positive (IHC score: $3+$ ). Of the three patients in the loss of HER2 expression group, two patients had a recurrence at 9.9-11.9 months after gastrectomy or liver resection (average: 10.9 months). One patient had skin metastasis 9.9 months after gastrectomy, and metastatic tumors were surgically removed. HER2 expression in skin metastasis was negative (IHC score: $0)$. Remaining patient in the loss of HER2 expression group had no recurrence for 24.1 months after gastrectomy.

\section{Discussion}

To the best of our knowledge, few studies have focused on the loss of HER2 expression in resected specimens collected from patients with advanced HER2-positive gastric cancer treated with trastuzumab-based chemotherapy. In the present study, we demonstrated that in three out of seven patients (42.9\%), HER2-positive tumor cells in resected specimens disappeared after trastuzumab-based chemotherapy. To date, several investigators have reported the loss of HER2 positivity in $29 \%-61 \%$ of biopsied tumors and $21 \%-42 \%$ of surgical specimens obtained from patients with gastric cancer, after trastuzumab-based chemotherapy (16-20). Consequently, these results are consistent with our findings showing loss of HER2 positivity (42.9\%).

The biological mechanism for the loss of HER2 positivity after trastuzumab-based chemotherapy remains unclear. However, several reasons for this loss of HER2 expression have been proposed in patients with advanced HER2-positive gastric cancer after trastuzumab-based chemotherapy. Firstly, intratumoral HER2 heterogeneity may be a key issue in understanding loss of HER2 positivity. When assessing HER2 expression in patients with gastric cancer, we should consider the possibility of falsenegative results when HER2 expression is detected via IHC. The incidence of heterogeneous HER2 overexpression was reported to be $69 \%-75 \%$ in patients with HER2-positive gastric cancer evaluated via IHC (21-23). Accordingly, a false-negative result for HER2 expression may be caused by assessing IHC in a portion of a tumor using biopsied specimens. As such, Tominaga et al. demonstrated that using five tumor biopsy specimens from 


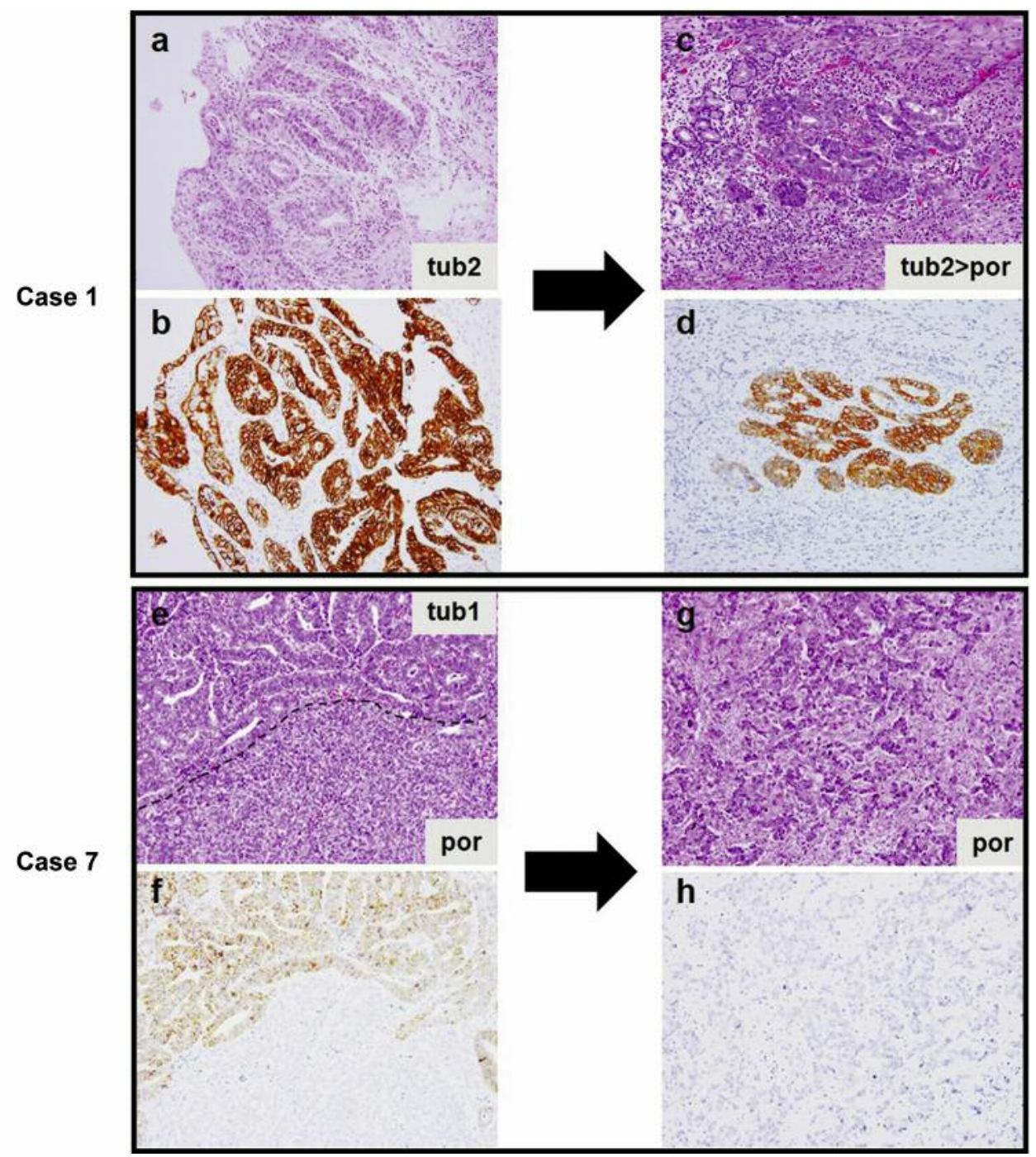

Figure 1. Representative staining for HER2 status. $(a, b)$ Biopsied specimens before chemotherapy and $(c, d)$ resected specimens after chemotherapy in the maintained HER2 expression group (Case 1). (a) Hematoxylin and eosin (HE) staining indicated a moderately differentiated tubular adenocarcinoma (tub2) (original magnification: $\times 200$ ). (b) HER2 immunohistochemistry (IHC) was scored as 3+ (original magnification $\times 200)$. (c) HE findings indicated a tub2 and poorly differentiated adenocarcinoma (por) (original magnification: $\times 200)$. (d) HER2 IHC was scored as 3+ (original magnification: $\times 200)$. $(e, f)$ Resected gastric specimen before trastuzumab-based chemotherapy and $(g, h)$ resected liver metastatic specimens after chemotherapy in the loss of HER2 expression group (Case 7). (e) HE staining indicated a mixed type of well differentiated tubular adenocarcinoma (tub1) and por (original magnification: $\times 200$ ). Tumor cells showing tub1 and por were identified in the upper and lower areas of the dotted line, respectively. (f) HER2 IHC findings indicated a strong basolateral membranous reactivity only in tumor cells with tub1; there was no membranous reactivity in any tumor cells with por. HER2 IHC was scored as $3+$ (original magnification: $\times 200$ ). $(\mathrm{g}) \mathrm{HE}$ findings indicated a por (original magnification: $\times 200$ ). (h) HER2 IHC findings indicated no membranous reactivity in any tumor cells. HER2 IHC was scored as 0 (original magnification: $\times 200$ ).

the proximal part of the tumor is advisable when performing HER2 testing in gastric cancer (24).

Secondly, the number of HER2 negative tumor cells may increase after trastuzumab-based chemotherapy. Recent studies in breast cancer patients have indicated that trastuzumab-based chemotherapy has a significant impact on loss of HER2 positivity, and have suggested that treatment-induced clonal selection, biological modification, and tumor heterogeneity could explain this impact $(25,26)$. HER2-positive gastric tumor cells have been shown to have higher chemosensitivity compared with HER2-negative gastric tumor cells (27). In the present study, only HER2-positive tumor cells with the dominant histological type disappeared in the loss of HER2 expression group, while HER2-negative tumor cells with a different histological type appeared. Moreover, the present study suggested that HER2-negative tumor cells after trastuzumab- 
based chemotherapy represent a dominant histological type because of low chemosensitivity. To the best of our knowledge, the present study is the first to examine histological tumor cells associated with chemoresistance as an important factor for loss of HER2 positivity after trastuzumab-based chemotherapy. Interestingly, Jordan et al. reported that oxidative stress or cytotoxic chemotherapy enhances the transition from HER2positive to HER2-negative in circulating tumor cells from breast cancer (28). These findings suggest that tumor cells with different chemosensitivity may enhance HER2 alteration from positive to negative after trastuzumab-based chemotherapy.

The third key issue is the effect of formalin fixation conditions. Saeki et al. reported that the use of fixatives other than $10 \%$ neutral buffered-formalin significantly reduce the HER2 overexpression rate (18). In the present study, neutral buffered-formalin at $10 \%$ and $20 \%$ was used for HER 2 IHC in six and one out of seven patients, respectively. In fact, the one patient that showed HER2-negative detection in HER2 IHC was assessed using $20 \%$ neutral buffered-formalin fixation conditions. Accordingly, higher formalin concentrations may reduce HER2 immunoreactivity. Additional research is needed to clarify the biological mechanism and clinical significance of HER2 loss related to HER2-targeted therapy in patients with advanced HER2-positive gastric cancer.

In the present study, we evaluated HER2 expression in recurrent sites of brain and skin obtained from two patients with disease recurrence after trastuzumab-based chemotherapy followed by gastrectomy. One patient showed HER2-positive status in all of his pre-therapeutic biopsied specimens, resected gastric specimens after trastuzumab-based chemotherapy, and recurrent brain specimens. Another patient showed HER2positive status in his biopsied sample and HER2-negative status in both resected primary gastric tumors and skin metastasis after treatment. These findings suggest that HER2 status could change even in recurrent sites after treatments.

Several reports have indicated that breast cancer patients showing loss of HER2 expression after trastuzumab-based chemotherapy have a lower relapse-free survival rate than those who maintained HER2 expression $(11,12)$. On the other hand, Seo et al. reported that loss of HER2 expression after trastuzumab-based chemotherapy was not significantly associated with worse progression in gastric cancer (16). Similarly, we did not observe a difference in relapse-free survival between the maintained HER 2 expression group and the loss of HER2 expression group (data not shown). These results may depend on differences in tumor biology between gastric cancer and breast cancer (22).

Our study had several limitations. This study was conducted on a small patient sample, in a single institution. Furthermore, various systemic chemotherapy regimens with trastuzumab before surgical treatments were used in these patients. Accordingly, further multicenter validation studies are warranted to confirm our results.
In conclusion, the present study demonstrated an alteration in HER2 expression after trastuzumab-based chemotherapy in patients with advanced HER2-positive gastric cancer. Since little is understood about the clinical impact of HER2 loss, reassessment of HER2 expression after trastuzumab-based chemotherapy is important to deliver appropriate therapy in patients with advanced HER2-positive gastric cancer.

\section{Conflicts of Interest}

The Authors have no conflicts of interest regarding this study.

\section{Authors' Contributions}

TK analyzed and interpreted the data and prepared the manuscript draft. DM, KO, MS, and SY enrolled the patients and collected the clinical data. TH and AT performed the pathological analysis. TA, YU, SI and KM participated in planning the study, the interpretation of clinical aspect and writing of manuscript. SN supervised the overall research work including planning the study, interpretation and drafting of the manuscript. All Authors read and approved this manuscript.

\section{References}

1 Ferlay J, Soerjomataram I, Dikshit R, Eser S, Mathers C, Rebelo M, Parkin DM, Forman D and Bray F: Cancer incidence and mortality worldwide: Sources, methods and major patterns in globocan 2012. Int J Cancer 136(5): E359-386, 2015. PMID: 25220842. DOI: $10.1002 / \mathrm{ijc} .29210$

2 Van Cutsem E, Sagaert X, Topal B, Haustermans K and Prenen H: Gastric cancer. Lancet 388(10060): 2654-2664, 2016. PMID: 27156933. DOI: $10.1016 / \mathrm{S} 0140-6736(16) 30354-3$

3 Fontana E and Smyth EC: Novel targets in the treatment of advanced gastric cancer: A perspective review. Ther Adv Med Oncol 8(2): 113125, 2016. PMID: 26929787. DOI: 10.1177/1758834015616935

4 Gravalos C and Jimeno A: Her2 in gastric cancer: A new prognostic factor and a novel therapeutic target. Ann Oncol 19(9): 1523-1529, 2008. PMID: 18441328. DOI: 10.1093/annonc/mdn169

5 Bang YJ, Van Cutsem E, Feyereislova A, Chung HC, Shen L, Sawaki A, Lordick F, Ohtsu A, Omuro Y, Satoh T, Aprile G, Kulikov E, Hill J, Lehle M, Ruschoff $J$ and Kang YK: Trastuzumab in combination with chemotherapy versus chemotherapy alone for treatment of her2-positive advanced gastric or gastro-oesophageal junction cancer (toga): A phase 3, openlabel, randomised controlled trial. Lancet 376(9742): 687-697, 2010. PMID: 20728210. DOI: 10.1016/S0140-6736(10)61121-X

6 Yamaguchi K, Yoshida K, Tanahashi T, Takahashi T, Matsuhashi $\mathrm{N}$, Tanaka Y, Tanabe K and Ohdan H: The long-term survival of stage IV gastric cancer patients with conversion therapy. Gastric Cancer 21(2): 315-323, 2018. PMID: 28616743. DOI: 10.1007/s 10120-017-0738-1

7 Sbitti Y, Essaidi I, Debbagh A, Kadiri H, Oukabli M, Moussaid Y, Slimani K, Fetohi M, Elkaoui H, Albouzidi A, Mahi M, Ali AA, Ichou $\mathrm{M}$ and Errihani $\mathrm{H}$ : Is there any advantage to combined trastuzumab and chemotherapy in perioperative setting her 2 neu positive localized gastric adenocarcinoma? World J Surg Oncol 9(112), 2011. PMID: 21955806. DOI: 10.1186/1477-7819-9-112 
8 Dong CX, Fu JF, Ye XY, Li XF, Zhong X and Yuan Y: Surgical resection of advanced gastric cancer following trastuzumab/ oxaliplatin/capecitabine combination therapy. World $\mathbf{J}$ Gastroenterol 20(34): 12355-12358, 2014. PMID: 25232273. DOI: $10.3748 /$ wjg.v20.i34.12355

9 Ikari N, Nakajima G, Taniguchi K, Sasagawa T, Narumiya K, Yamada T, Kudo K, Kitamura Y, Hayashi K, Nishikawa T and Yamamoto M: Her2-positive gastric cancer with paraaortic nodal metastasis successfully resected after chemotherapy with trastuzumab: A case report. Anticancer Res 34(2): 867-872, 2014. PMID: 24511024.

10 Ishimine Y, Goto A, Watanabe Y, Yajima H, Nakagaki S, Yabana T, Adachi T, Kondo Y and Kasai K: Loss of her2 positivity after trastuzumab in her2-positive gastric cancer: Is change in her2 status significantly frequent? Case Rep Gastrointest Med 2015: 132030, 2015. PMID: 25893119. DOI: 10.1155/2015/132030

11 Guarneri V, Dieci MV, Barbieri E, Piacentini F, Omarini C, Ficarra G, Bettelli S and Conte PF: Loss of her2 positivity and prognosis after neoadjuvant therapy in her2-positive breast cancer patients. Ann Oncol 24(12): 2990-2994, 2013. PMID: 24013581. DOI: $10.1093 /$ annonc/mdt364

12 Mittendorf EA, Wu Y, Scaltriti M, Meric-Bernstam F, Hunt KK, Dawood S, Esteva FJ, Buzdar AU, Chen H, Eksambi S, Hortobagyi GN, Baselga $\mathrm{J}$ and Gonzalez-Angulo AM: Loss of her2 amplification following trastuzumab-based neoadjuvant systemic therapy and survival outcomes. Clin Cancer Res 15(23): 7381-7388, 2009. PMID: 19920100. DOI: 10.1158/1078-0432.CCR-09-1735

13 Edge SB and Compton CC: The american joint committee on cancer: The 7th edition of the ajcc cancer staging manual and the future of TNM. Ann Surg Oncol 17(6): 1471-1474, 2010. PMID: 20180029. DOI: 10.1245/s10434-010-0985-4

14 Japanese classification of gastric carcinoma: 3rd english edition. Gastric Cancer 14(2): 101-112, 2011. PMID: 21573743. DOI: 10.1007/s10120-011-0041-5

15 Hofmann M, Stoss O, Shi D, Buttner R, van de Vijver M, Kim W, Ochiai A, Ruschoff $\mathrm{J}$ and Henkel T: Assessment of a her2 scoring system for gastric cancer: Results from a validation study. Histopathology 52(7): 797-805, 2008. PMID: 18422971. DOI: $10.1111 / \mathrm{j} .1365-2559.2008 .03028 . \mathrm{x}$

16 Seo S, Ryu MH, Park YS, Ahn JY, Park Y, Park SR, Ryoo BY, Lee GH, Jung HY and Kang YK: Loss of her2 positivity after anti-her2 chemotherapy in her2-positive gastric cancer patients: Results of the gastric cancer her2 reassessment study 3 (gasther3). Gastric Cancer, 2018. PMID: 30386954. DOI: 10.1007/s10120-018-0891-1

17 Pietrantonio F, Caporale M, Morano F, Scartozzi M, Gloghini A, De Vita F, Giommoni E, Fornaro L, Aprile G, Melisi D, Berenato R, Mennitto A, Volpi CC, Laterza MM, Pusceddu V, Antonuzzo L, Vasile E, Ongaro E, Simionato F, de Braud F, Torri V and Di Bartolomeo M: Her2 loss in her2-positive gastric or gastroesophageal cancer after trastuzumab therapy: Implication for further clinical research. Int J Cancer 139(12): 2859-2864, 2016. PMID: 27578417. DOI: 10.1002/ijc.30408

18 Saeki H, Oki E, Kashiwada T, Arigami T, Makiyama A, Iwatsuki M, Narita Y, Satake H, Matsuda Y, Sonoda H, Shimokawa M and Maehara Y: Re-evaluation of her2 status in patients with her2-positive advanced or recurrent gastric cancer refractory to trastuzumab (kscc1604). Eur J Cancer 105: 41-49, 2018. PMID: 30391779. DOI: 10.1016/j.ejca.2018.09.024

19 Watson S, Validire P, Cervera P, Zorkani N, Scriva A, Lemay F, Tournigand C, Perniceni T, Garcia ML, Bennamoun M, Paye F and Louvet $\mathrm{C}$ : Combined her2 analysis of biopsies and surgical specimens to optimize detection of trastuzumab-eligible patients in eso-gastric adenocarcinoma: A gercor study. Ann Oncol 24(12): 3035-3039, 2013. PMID: 24114855. DOI: 10.1093/annonc/mdt393

20 Hedner C, Borg D, Nodin B, Karnevi E, Jirstrom K and Eberhard J: Expression and prognostic significance of human epidermal growth factor receptors 1,2 and 3 in oesophageal and gastric adenocarcinomas preneoadjuvant and postneoadjuvant treatment. J Clin Pathol 71(5): 451-462, 2018. PMID: 29138285. DOI: $10.1136 /$ jclinpath-2017-204774

21 Kurokawa Y, Matsuura N, Kimura Y, Adachi S, Fujita J, Imamura H, Kobayashi K, Yokoyama Y, Shaker MN, Takiguchi $\mathrm{S}$, Mori $\mathrm{M}$ and Doki Y: Multicenter large-scale study of prognostic impact of her2 expression in patients with resectable gastric cancer. Gastric Cancer 18(4): 691-697, 2015. PMID: 25224659. DOI: 10.1007/s10120-014-0430-7

22 Lee HE, Park KU, Yoo SB, Nam SK, Park do J, Kim HH and Lee HS: Clinical significance of intratumoral her2 heterogeneity in gastric cancer. Eur J Cancer 49(6): 1448-1457, 2013. PMID: 23146959. DOI: $10.1016 /$ j.ejca.2012.10.018

23 Motoshima S, Yonemoto K, Kamei H, Morita M and Yamaguchi $\mathrm{R}$ : Prognostic implications of her2 heterogeneity in gastric cancer. Oncotarget 9(10): 9262-9272, 2018. PMID: 29507688. DOI: $10.18632 /$ oncotarget.24265

24 Tominaga N, Gotoda T, Hara M, Hale MD, Tsuchiya T, Matsubayashi J, Kono S, Kusano C, Itoi T, Fujimoto K, Moriyasu $\mathrm{F}$ and Grabsch HI: Five biopsy specimens from the proximal part of the tumor reliably determine her 2 protein expression status in gastric cancer. Gastric Cancer, 2015. PMID: 25987463. DOI: 10.1007/s 10120-015-0502-3

25 Wang RX, Chen S, Jin X, Chen CM and Shao ZM: Weekly paclitaxel plus carboplatin with or without trastuzumab as neoadjuvant chemotherapy for her2-positive breast cancer: Loss of her2 amplification and its impact on response and prognosis. Breast Cancer Res Treat 161(2): 259-267, 2017. PMID: 27885439. DOI: $10.1007 / \mathrm{s} 10549-016-4064-9$

26 Niikura N, Tomotaki A, Miyata H, Iwamoto T, Kawai M, Anan K, Hayashi N, Aogi K, Ishida T, Masuoka H, Iijima K, Masuda S, Tsugawa K, Kinoshita T, Nakamura S and Tokuda Y: Changes in tumor expression of her2 and hormone receptors status after neoadjuvant chemotherapy in 21755 patients from the japanese breast cancer registry. Ann Oncol 27(3): 480-487, 2016. PMID: 26704052. DOI: 10.1093/annonc/mdv611

27 Lordick F, Kang YK, Chung HC, Salman P, Oh SC, Bodoky G, Kurteva G, Volovat C, Moiseyenko VM, Gorbunova V, Park JO, Sawaki A, Celik I, Gotte H, Melezinkova H and Moehler M: Capecitabine and cisplatin with or without cetuximab for patients with previously untreated advanced gastric cancer (expand): A randomised, open-label phase 3 trial. Lancet Oncol 14(6): 490-499, 2013. PMID: 23594786. DOI: 10.1016/S1470-2045(13)70102-5

28 Jordan NV, Bardia A, Wittner BS, Benes C, Ligorio M, Zheng Y, Yu M, Sundaresan TK, Licausi JA, Desai R, O'Keefe RM, Ebright RY, Boukhali M, Sil S, Onozato ML, Iafrate AJ, Kapur R, Sgroi D, Ting DT, Toner M, Ramaswamy S, Haas W, Maheswaran S and Haber DA: Her2 expression identifies dynamic functional states within circulating breast cancer cells. Nature 537(7618): 102-106, 2016. PMID: 27556950. DOI: 10.1038/nature19328

Received November 16, 2019

Revised November 24, 2019 Accepted November 28, 2019 